\title{
Prognostic Role of Serum Lactate Dehydrogenase Beyond Initial Diagnosis: A Retrospective Analysis of Patients with Diffuse Large B Cell Lymphoma
}

\author{
Junshik Hong ${ }^{a}$ Hyun-Hwa Yoon ${ }^{a}$ Hee Kyung Ahn ${ }^{a}$ Sun Jin Sym ${ }^{a}$ Jinny Park ${ }^{a}$ \\ Pil-Whan Park ${ }^{\mathrm{b}}$ Jeong Yeal Ahn ${ }^{\mathrm{b}}$ Sanghui Park ${ }^{\mathrm{c}}$ Eun Kyung Cho ${ }^{\mathrm{a}}$ \\ Dong Bok Shin ${ }^{a}$ Jae Hoon Lee ${ }^{a}$ \\ Departments of ${ }^{\mathrm{a}}$ Internal Medicine and ${ }^{\mathrm{b}}$ Laboratory Medicine, Gachon University School of Medicine, Incheon, and \\ 'Department of Pathology, Ewha Womans University School of Medicine, Seoul, Republic of Korea
}

\section{Key Words \\ Lactate dehydrogenase $\cdot$ Non-Hodgkin lymphoma . \\ Diffuse large B cell lymphoma . Prognosis}

\begin{abstract}
Background/Aims: Baseline serum lactate dehydrogenase (LDH) level is a well-known prognostic factor in patients with non-Hodgkin's lymphoma; however, its role beyond initial diagnosis has not yet been defined. Methods: This study was conducted as a retrospective analysis of patients with diffuse large $B$ cell lymphoma (DLBCL) treated with R-CHOP21, who had undergone regular checks for $\mathrm{LDH}$ during immunochemotherapy $(n=119)$ and during the posttreatment followup period after complete remission $(C R ; n=100)$. The 119 patients were classified into 4 groups according to their baseline and change in LDH level during treatment, and an analysis of tumor response and survival was performed. The value of $\mathrm{LDH}$ as a predictor for relapse was evaluated among the patients with regular follow-up visits after achieving CR. Results: An increased LDH level during immunochemotherapy had no impact on tumor response or survival, and only the LDH status 'before' treatment was a prognostic marker. The sensitivity, specificity, positive predictive value and negative predictive value of serum $\mathrm{LDH}$ for detecting relapse afterCRwere 47.4,86.5,9.3 and 98.3\%, respectively.Conclusion:
\end{abstract}

The measurement of LDH level beyond initial diagnosis has no clear benefit in predicting disease progression or relapse in patients with DLBCL treated with R-CHOP21.

Copyright $\odot 2013$ S. Karger AG, Basel

\section{Introduction}

Lactate dehydrogenase (LDH) is an enzyme found in almost all tissues of living organisms, including plants and animals [1]. In humans, it is a surrogate for tissue breakdown due to various causes, including muscle injury, hemolytic anemia, acute coronary syndrome, diverse infectious or inflammatory conditions and certain malignancies. The elevation of LDH in the blood is a relatively nonspecific phenomenon; however, it has been recognized as a tumor marker, as it reflects tumor burden and cellular turnover in several aggressive malignancies, including germ cell tumors [2], sarcomas [3-4] and nonHodgkin lymphoma (NHL) [5-6].

Patients with elevated serum LDH at the time of initial diagnosis have inferior survival outcomes, compared to those with normal LDH levels. Therefore, LDH has been a component of the International Prognostic Index (IPI) [5], a clinical tool for predicting the prognosis of patients with aggressive NHL. However, the role of serum LDH

\section{KARGER}

E-Mail karger@karger.com

www.karger.com/aha
(C) 2013 S. Karger AG, Basel

0001-5792/13/1304-0305\$38.00/0
Jae Hoon Lee, MD, $\mathrm{PhD}$

Department of Internal Medicine, Gachon University School of Medicine Gachon University Gil Medical Center, 21 Namdongdae-ro 774-gil

Namdong-gu, Incheon, 405-760 (Republic of Korea)

E-Mail jhlee@ gilhospital.com 
beyond initial diagnosis, i.e. during active chemotherapy and the posttreatment follow-up period, has not yet been well defined. For physicians, an increase in LDH or the maintenance of elevation above the normal range during or after active treatment may indicate poor treatment outcome and early disease relapse/progression. The European Society for Medical Oncology has recommended determining LDH levels at 3, 6, 12, and 24 months after completing chemotherapy via follow-up visits for patients with diffuse large B cell lymphoma (DLBCL) [7]. However, this is based on the custom of clinical practice rather than on established evidence. Although no other objective evidence exists, elevated LDH during posttherapy follow-up periods may also cause anxiety for patients and doctors due to the fear of disease relapse.

In this study, we conducted a retrospective analysis of the prognostic role of serum LDH during and after the treatment of patients within a homogeneous disease population of aggressive NHL, DLBCL undergoing a standard treatment, three weekly treatments of rituximab with cyclophosphamide, doxorubicin, vincristine and prednisolone (R-CHOP21) immunochemotherapy.

\section{Methods}

\section{Patients and Treatment}

The inclusion criteria for the patients in this study were: (1) a histologic diagnosis of DLBCL (according to the World Health Organization criteria) between May 2004 and December 2012 at a single institution, the Gachon University Gil Hospital (GUGH), (2) treatment with and completion of at least three cycles of RCHOP21 immunochemotherapy, (3) age $\geq 20$ years and (4) a complete set of clinical and laboratory data for use in the retrospective analysis. Patients with DLBCL transformed from an indolent lymphoma, primary central nervous system DLBCL or HIV-associated DLBCL were not included in the analysis.

Among the patients who fulfilled the inclusion criteria, patients who underwent LDH testing before every cycle of R-CHOP21 therapy were selected and included in group DIC (i.e. during immunochemotherapy). The LDH level was tested no more than 5 days before day 1 of the next cycle, and patients who lacked two or more reports of $\mathrm{LDH}$ during treatment were excluded from group DIC. The patients were classified according to the overall trend of their $\mathrm{LDH}$ level during treatment periods as follows: (1) elevated $\mathrm{LDH}$ at the initial diagnosis with a gradual decrease and normalization with continuation of R-CHOP21 $\left(\mathrm{LDH}_{\uparrow / \leftrightarrow}\right)$, (2) elevated LDH at the initial diagnosis and persistent elevation during and just after treatment $\left(\mathrm{LDH}_{\uparrow / \uparrow}\right),(3)$ nonelevated LDH before R-CHOP21 and maintenance of $\mathrm{LDH}$ within the normal range $\left(\mathrm{LDH}_{\leftrightarrow / \leftrightarrow}\right)$ and $(4)$ nonelevated $\mathrm{LDH}$ at the time of diagnosis, but elevated $\mathrm{LDH}$ during treatment $\left(\mathrm{LDH}_{\leftrightarrow / \uparrow}\right)$. A single or two nonconsecutive episodes of change in $\mathrm{LDH}$ (either from normal range to elevated range or vice versa) were not considered to be a true change in LDH status. Only gradual, consecutive changes in LDH levels were classified as genuine changes.
Among the patients who achieved a metabolic complete response/complete remission (CR), patients who underwent LDH testing before each follow-up visit were selected and included in group AIC (i.e. after immunochemotherapy). An evaluation of serum $\mathrm{LDH}$ was performed within 7 days of the follow-up visit. $\mathrm{Pa}$ tients who had two or more omissions of LDH testing and/or compatible imaging with follow-up visits were excluded from group AIC. The value of $\mathrm{LDH}$ as a predictor for relapse was evaluated.

This study was reviewed and approved by the institutional review board of GUGH (approval number: GIRBA2781-2012).

\section{Treatment and Tumor Evaluation}

R-CHOP21 immunochemotherapy was administered using doses reported in previous studies [8-9]. Patients with limitedstage DLBCL were treated with either 3-4 cycles of R-CHOP21 followed by involved field radiotherapy, or 6-8 cycles of RCHOP21 with or without radiotherapy. Patients with advancedstage DLBCL received 6-8 cycles of $\mathrm{R}-\mathrm{CHOP} 21$ with or without radiotherapy.

During R-CHOP21 therapy, all patients underwent computed tomography (CT) of the lymphoma-involved area every 2-4 cycles for the evaluation of tumor status. Among patients who had received 6-8 cycles of R-CHOP21, an interim fludeoxyglucose-positron emission tomography (FDG-PET)/CT was performed at the end of cycles 2-4 of R-CHOP21. Before every cycle, blood tests including complete blood cell count (CBC), serum chemistry and LDH were performed to confirm the recovery of $\mathrm{CBC}$ and adequate organ function to initiate the next cycle. After completing immunochemotherapy, the tumor responses were evaluated according to the Revised International Workshop criteria [10]. The patients were evaluated every 3 months during the first 2 years, then every $4-6$ months for the next 3 years and annually thereafter, in order to monitor the relapse of DLBCL. CT scanning of the area of initial tumor involvement, with or without additional CT scanning of the chest/abdominopelvic area according to the physician's decision, was performed during every regular follow-up visit, along with noting the medical history, a physical examination and blood tests including $\mathrm{CBC}$, serum chemistry and LDH. The evaluation of the follow-up FDG$\mathrm{PET} / \mathrm{CT}$ was performed according to coverage provided by the Korean National Health Insurance: twice in 1 year for the first 2 years after chemotherapy and once every 2 years thereafter. Regular CT scanning was omitted for patients who underwent evaluation by FDG-PET/CT. For patients with a potential for relapse or progression of DLBCL, FDG-PET/CT and/or CT scan of additional areas was performed. In principle, the pathologic diagnosis to confirm relapse and initiate salvage chemotherapy was mandatory.

\section{Analysis of Serum LDH}

Serum LDH levels were measured using an autoanalyzer (ADVIA 2400: SIEMENS Healthcare Diagnostics, Tarrytown, N.Y., USA), according to the manufacturer's instructions. The upper limit of the normal value of serum LDH for the GUGH was 485 U/liter. The patient's history of infection (fever with clinical and/or laboratory evidence of infection), trauma and use of granulocytecolony stimulating factor (G-CSF) were retrospectively reviewed. Hemolyses during blood sampling, which had been reported in the electronic medical record system of GUGH, were reviewed along with the LDH results. Serum LDH with hemolysis, infection, any trauma and recent (within 5 days) administration of G-CSF were regarded as 'no check', and were excluded from the analysis. 
Table 1. Patient characteristics

\begin{tabular}{|c|c|c|c|c|c|c|}
\hline \multirow[t]{3}{*}{ Characteristics } & \multicolumn{6}{|c|}{ Analysis set } \\
\hline & \multicolumn{2}{|c|}{ all patients $(n=143)$} & \multicolumn{2}{|c|}{ group DIC $(n=119)$} & \multicolumn{2}{|c|}{ group AIC $(n=100)$} \\
\hline & $\mathrm{n}$ & $\%$ & $\mathrm{n}$ & $\%$ & $\mathrm{n}$ & $\%$ \\
\hline \multicolumn{7}{|l|}{ Gender } \\
\hline Male & 70 & 49.0 & 60 & 50.4 & 58 & 58.0 \\
\hline Female & 73 & 51.0 & 59 & 49.6 & 42 & 42.0 \\
\hline$>60$ & 69 & 48.3 & 52 & 43.7 & 59 & 59.0 \\
\hline$\leq 60$ & 74 & 51.7 & 67 & 56.3 & 41 & 41.0 \\
\hline \multicolumn{7}{|l|}{$\mathrm{LDH}$} \\
\hline Not elevated & 81 & 56.6 & 67 & 56.3 & 60 & 60.0 \\
\hline Elevated & 62 & 43.4 & 52 & 43.7 & 40 & 40.0 \\
\hline \multicolumn{7}{|l|}{ ECOG performance status } \\
\hline III or IV & 67 & 46.9 & 53 & 44.5 & 42 & 42.0 \\
\hline \multicolumn{7}{|l|}{ Extranodal lesion } \\
\hline 0 or 1 site & 113 & 79.0 & 94 & 79.0 & 80 & 80.0 \\
\hline$\geq 2$ sites & 30 & 21.0 & 25 & 21.0 & 20 & 20.0 \\
\hline \multicolumn{7}{|l|}{ Standard IPI } \\
\hline Low & 66 & 46.2 & 59 & 49.6 & 54 & 54.0 \\
\hline Low-intermediate & 34 & 23.8 & 28 & 23.5 & 23 & 23.0 \\
\hline High-intermediate & 22 & 15.4 & 18 & 15.1 & 15 & 15.0 \\
\hline High & 21 & 14.7 & 14 & 11.8 & 8 & 8.0 \\
\hline \multicolumn{7}{|l|}{ Hans classification } \\
\hline GCB type & 40 & 28.0 & 36 & 30.3 & 31 & 31.0 \\
\hline Non-GCB type & 59 & 41.3 & 52 & 43.7 & 41 & 41.0 \\
\hline \multicolumn{7}{|l|}{ Survival estimation } \\
\hline Median follow-up duration, months & 18.8 & & 22.0 & & 31.6 & \\
\hline Two-year EFS, \% & 70.1 & & 76.4 & & 81.8 & \\
\hline Two-year OS, \% & 77.4 & & 82.7 & & 90.5 & \\
\hline
\end{tabular}

ECOG $=$ Eastern cooperative oncology group; $\mathrm{GCB}=$ germinal center B cell .

\section{Statistical Analysis}

Event-free survival (EFS) was measured from the time of treatment initiation to any treatment failure including disease progression, or discontinuation of treatment for any reason (e.g. disease progression, toxicity, patient preference or death). Overall survival (OS) was defined as survival free of death from any cause. Survival analysis was performed using the Kaplan-Meier method and the log-rank test was used for comparison. Continuous biologic variables were dichotomized and the Fisher exact test or the $\chi^{2}$ test were used as appropriate. All values were 2 -sided and statistical significance was accepted at the level of $\mathrm{p}<0.05$.

LDH in Diffuse Large B Cell Lymphoma

\section{Results}

\section{Patient Characteristics and Overall Treatment}

Outcomes

A total of 143 patients satisfied the inclusion criteria. Group DIC consisted of 119 patients and group AIC consisted of 100. A summary of the baseline characteristics of the entire patient population and the groups is shown in table 1. 
Tumor responses after R-CHOP21 therapy were as follows: 121 patients (84.6\%) achieved a CR, 17 (11.9\%) showed a partial response and $8(5.6 \%)$ could not be evaluated due to treatment-related mortality $(n=3)$, not undergoing FDG-PET/CT according to the Revised International Workshop criteria (CT scan only, $\mathrm{n}=3$ ) and refusal by the patient $(n=2)$. During a median follow-up duration of 18.8 months, the 2-year EFS and OS were 70.2 and $77.4 \%$, respectively. Elevated $\mathrm{LDH}$ at baseline was a strong poor prognostic factor for EFS [hazard ratio (HR) $2.88,95 \%$ confidence interval $(\mathrm{CI}) 1.50-5.52 ; \mathrm{p}=0.002$ ] and OS (HR 2.78, 95\% CI 1.36-5.71; $\mathrm{p}=0.005$ ).

\section{Serum LDH during Immunochemotherapy}

Among 119 patients in group DIC, 627 cycles of RCHOP21 immunochemotherapy were administered, and 566 cycles $(90.3 \%)$ were included in the analysis of serum LDH; 34 cycles were excluded due to omission of LDH testing, 14 cycles due to hemolysis of blood sample, 7 cycles due to G-CSF use and 6 cycles because patients had concomitant systemic infections or inflammation.

Twenty-six of 119 patients (21.8\%) were classified as $\mathrm{LDH}_{\uparrow / \leftrightarrow}$ (LDH elevation did not occur in 12 patients, and 14 had an episodic elevation; however, their overall trends showed normalization of LDH). Twenty-seven patients (22.7\%) were classified as $\mathrm{LDH}_{\uparrow / \uparrow}$ (the $\mathrm{LDH}$ level never normalized in 5 patients, whereas 22 experienced one or two occurrences of transient normalization; however, overall trends indicated persistent elevation, and they completed therapy with elevated LDH). Fifty-three patients $(44.5 \%)$ were classified as $\mathrm{LDH}_{\leftrightarrow / \leftrightarrow}$ (24 patients maintained a normal LDH level without elevation, and 29 experienced sporadic elevation of $\mathrm{LDH}$ ). Thirteen patients $\left(10.9 \%\right.$ ) were classified as $\mathrm{LDH}_{\leftrightarrow / \uparrow}$ (all 13 patients experienced a gradual increase in LDH during immunochemotherapy and, once elevated, the LDH did not become normalized).

A total of 116 patients were evaluable for tumor response, and most patients achieved CR (110 patients; $92.4 \%)$. The LDH status both before $(\mathrm{p}=0.693)$ and during treatment $(\mathrm{p}=0.392)$ had no influence on tumor response after treatment (table 2).

Elevated LDH during R-CHOP21 therapy was not a poor prognostic factor; no significant differences of EFS and OS were observed between the $\mathrm{LDH}_{\uparrow / \leftrightarrow}$ and $\mathrm{LDH}_{\uparrow / \uparrow}$ groups, nor did an increase in LDH have any influence on EFS and OS in either group (fig. 1). No disease progression during treatment with $\mathrm{R}-\mathrm{CHOP} 21$ was reported among 40 patients with a gradual elevation of $\mathrm{LDH}$
Table 2. Tumor response after R-CHOP 21 therapy according to serum LDH status during immunochemotherapy

\begin{tabular}{lccc}
\hline & \multicolumn{2}{l}{$\begin{array}{l}\text { Response by the Revised International } \\
\text { Workshop criteria }\end{array}$} & Total \\
\cline { 2 - 3 } & CR & No CR (partial remission) & \\
\hline $\mathrm{LDH}_{\uparrow / \leftrightarrow}$ & 24 & 1 & 25 \\
$\mathrm{LDH}_{\uparrow / \uparrow}$ & 22 & 3 & 25 \\
$\mathrm{LDH}_{\leftrightarrow / \leftrightarrow}$ & 51 & 2 & 53 \\
$\mathrm{LDH}_{\leftrightarrow}$ & 13 & 0 & 13 \\
\hline Total & 110 & 6 & 116 \\
\hline
\end{tabular}

$\left(\mathrm{LDH}_{\uparrow / \uparrow}\right.$ and $\left.\mathrm{LDH}_{\leftrightarrow / \uparrow}\right)$ during immunochemotherapy. Of the patients in group DIC, we selected 52 who presented with elevated $\mathrm{LDH}$ at diagnosis and analyzed the prognostic value of a decrease in $\mathrm{LDH}$ after the 1-2 cycles of $\mathrm{R}-\mathrm{CHOP} 21$ (45 patients experienced decreases in $\mathrm{LDH}$, and 7 did not). No differences were observed in 2-year EFS (68.8\% for 45 patients vs. $68.6 \%$ for 7 patients, $\mathrm{p}=$ $0.827)$ and $O S(p=0.950)$.

\section{Serum LDH during Postimmunochemotherapy Follow-Up}

A total of 100 patients achieved CR after R-CHOP21 therapy and had sufficient data on serum LDH for inclusion in group AIC; among these, 671 follow-up visit events with appropriate serum LDH results were collected. An analysis of correlation between disease relapse and $\mathrm{LDH}$ elevation during posttreatment surveillance was performed.

Sensitivity, specificity, positive predictive value (PPV) and negative predictive value (NPV) of group AIC and high-risk patients by standard IPI are shown in table 3. Serum LDH levels indicated high specificity and NPV; however, its sensitivity was modest, and PPV was poor. We also used higher cut-off points for increased LDH in the analysis of group AIC. At a cut-off of $1.5 \times$ the upper limit of normal (ULN; 485 U/liter), the sensitivity, specificity, PPV and NPV were 21.1, 96.2, 13.8 and 97.7\%, respectively. When we used $2 \times$ ULN, the results were 10.5 , 97.1, 9.5 and $97.4 \%$, respectively.

Among the patients in group AIC, the role of serum LDH in 23 patients with IPI scores $\geq 3$ was analyzed separately for evaluation of the diagnostic usefulness of $\mathrm{LDH}$ in patients at a high-risk for relapse (table 3).

Twelve episodes of consecutive elevation of LDH were reported among 10 of the 100 patients; in 5 patients, consecutive elevation heralded tumor relapse. 


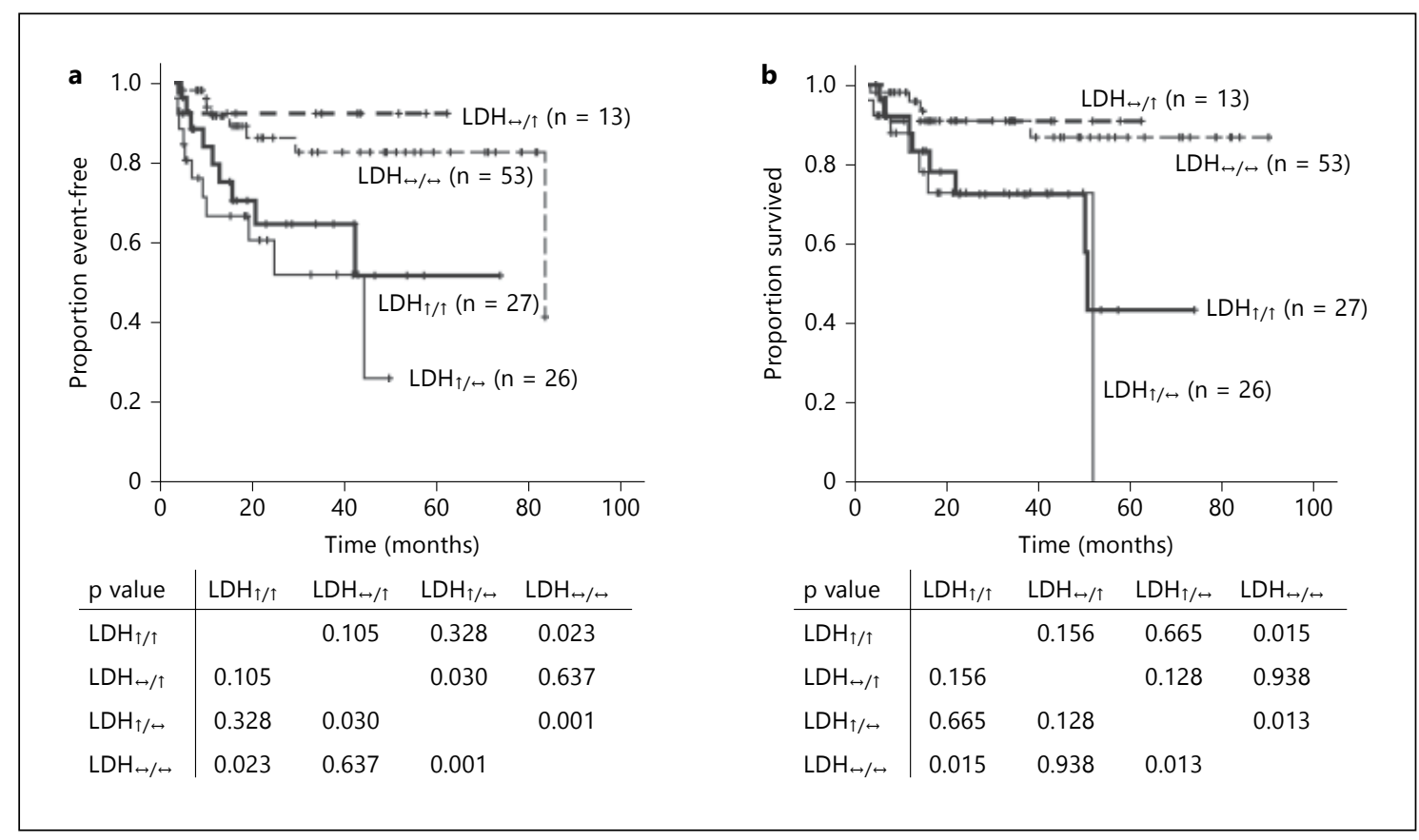

Fig. 1. Kaplan-Meier curve of EFS (a) and OS (b) according to serum LDH status during R-CHOP21 immunochemotherapy.

Table 3. Serum LDH for monitoring of disease relapse after immunochemotherapy

\begin{tabular}{|c|c|c|c|c|c|c|}
\hline & \multicolumn{3}{|c|}{$\begin{array}{l}\text { Diagnostic test evaluation for entire } \\
\text { population ( } 671 \text { visits, } n=100)\end{array}$} & \multicolumn{3}{|c|}{$\begin{array}{l}\text { Diagnostic test evaluation for high-risk } \\
\text { patients ( } 115 \text { visits, } n=23)\end{array}$} \\
\hline & relapse, no & relapse, yes & totals & relapse, no & relapse, yes & totals \\
\hline LDH elevated & 88 & 9 & 97 & 27 & 5 & 32 \\
\hline LDH not elevated & 564 & 10 & 574 & 81 & 2 & 83 \\
\hline Totals & 652 & 19 & 671 & 108 & 7 & 115 \\
\hline Sensitivity (95\% CI) & \multicolumn{3}{|c|}{$47.4 \%(24.5-71.0)$} & \multicolumn{3}{|c|}{$71.4 \%$ (35.9-91.8) } \\
\hline Specificity (95\% CI) & \multicolumn{3}{|c|}{$86.5 \%(83.6-89.0)$} & \multicolumn{3}{|c|}{$75.0 \%(66.1-82.2)$} \\
\hline PPV (95\% CI) & \multicolumn{3}{|c|}{$9.3 \%(4.3-16.9)$} & \multicolumn{3}{|c|}{$15.6 \%(6.9-31.8)$} \\
\hline NPV (95\% CI) & \multicolumn{3}{|c|}{$98.3 \%(96.8-99.2)$} & \multicolumn{3}{|c|}{$97.6 \%(91.6-99.3)$} \\
\hline
\end{tabular}

\section{Discussion}

In this study, the elevation of serum LDH during immunochemotherapy failed to predict poor treatment outcomes. In addition, due mainly to a high rate of falsepositive results, follow-up testing of LDH had limited value for monitoring lymphoma relapse among patients who achieved CR.

Currently, testing LDH levels during the period of immunochemotherapy for predicting tumor volume reduc- tion, as we have previously performed in several solid tumors with chorioembryonic antigen or alpha-fetoprotein, appears to be of no value in patients with DLBCL. More than half of the patients in group DIC (78/119; $65.5 \%)$ exhibited $\geq 1$ report of fluctuation in serum $\mathrm{LDH}$ levels during treatment, although most of them achieved CR. Therefore, we can assume that many factors can influence changes in serum LDH during immunochemotherapy. Even 13 patients (10.9\%) with normal LDH before treatment experienced gradual increases in $\mathrm{LDH}$ 
$\left(\mathrm{LDH}_{\leftrightarrow / \uparrow}\right)$ while undergoing R-CHOP21 therapy. However, they did not experience inferior EFS and OS compared to patients with $\mathrm{LDH}_{\leftrightarrow / \leftrightarrow}$. In addition, patients with $\mathrm{LDH}_{\leftrightarrow / \uparrow}$ experienced a significantly superior EFS ( $\mathrm{p}=$ $0.003)$ and a tendency of improved OS ( $p=0.128)$ compared to patients with $\mathrm{LDH}_{\uparrow / \leftrightarrow}$. These results suggest that the LDH status 'before' treatment, rather than 'during' treatment, is a true prognostic marker. A variety of responses by the human body to immunochemotherapy, such as inflammatory processes resulting from the immunochemotherapy itself, or a combined minor infection without fever resulting from immunosuppression, may contribute to such a nonspecific elevation of serum $\mathrm{LDH}$ during treatment.

Ten out of 19 patients who achieved CR after RCHOP21 therapy experienced a relapse of lymphoma without an increase of LDH after CR. Although serum $\mathrm{LDH}$ test results demonstrated relatively high specificity and NPV, the role of serum LDH as an ideal surveillance marker for relapse is not supported by other parameters. When we applied higher cut-off points for LDH to the analysis, despite a modest improvement in specificity, sensitivity and PPV were still too low for $\mathrm{LDH}$ to be a good diagnostic test. Weeks et al. [11] reported on a retrospective evaluation of the value of follow-up procedures in 139 patients with large-cell lymphoma who achieved CR after a high or moderate dose of methotrexate-based multi-agent chemotherapy. Despite the performance of a variety of posttherapy studies, including physical examination, $\mathrm{CBC}$, serum chemistry, serum $\mathrm{LDH}$, chest radiograph, gallium scan, CT scanning, ultrasound and bone marrow biopsy, the majority of relapses were detected during unscheduled visits for symptomatic disease (32 of 35, 91\%). Similar to our results, they reported that serum LDH demonstrated a sensitivity of $42 \%$ and a specificity of $85 \%$. They concluded that $\mathrm{LDH}$ was a successful parameter for detecting preclinical relapse of large-cell lymphoma; however, because their result demonstrated a relatively low sensitivity, the usefulness of serum LDH is doubtful.

In a recent retrospective study by El-Sharkawi et al. [12] evaluating the role of serum LDH in monitoring relapse $(n=102)$ in patients with DLBCL treated with rituximab-based immunochemotherapy, the serum LDH level was evaluated every 3 months during the first year, then every 4-6 months for the next 4 years and then annually. For patients with a relapse of DLBCL, the predictive value of $\mathrm{LDH}$ elevation for the 6 months preceding the relapse was evaluated. They reported sensitivity, specificity, PPV and NPV of $69,39,14$ and 90\%, respectively. We applied a different method for the analysis: each follow-up visit with LDH testing was a separate event. We thought that using this approach would allow us to avoid the risk of a high false-positive rate, because for some relapsed patients, relapse may not have been a gradual process lasting as long as even 6 months. Therefore, there may be no relationship between the relapse of DLBCL and an elevation of $\mathrm{LDH}$ at 3 or 6 months before the relapse. However, our methods may also have a bias toward those with the longest follow-up period, particularly those who have not relapsed, or if someone has an unexplained persistently high LDH. Both methods demonstrated the limitation of disease surveillance using LDH.

The treatment outcomes for patients with high-grade lymphoma differ substantially according to the IPI score. Therefore, the strategy of posttreatment monitoring could also differ according to the risk of relapse. In the analysis of LDH limited to patients with high or highintermediate risk by standard IPI, the problem of a high false-positive rate was not solved.

In this study, out of 10 patients, 12 events of serial LDH elevation during surveillance periods were reported. Five of these patients had experienced disease relapse at the time of the second or third elevation of LDH; all 5 were asymptomatic with a normal CT scan result at the time of the first LDH elevation. Then, at the time of subsequent LDH elevation (2-5 months after the first episode of LDH elevation), the patients all had symptoms that increased the possibility of disease relapse, and they were diagnosed as relapsed DLBCL. We can assume that consecutive elevations of LDH may have more diagnostic value than a single episode. However, we must use caution when interpreting the results, because the numbers of patients analyzed were too small to conclude that $\mathrm{LDH}$ testing should be repeated after every episode of LDH elevation.

Although routine scanning is generally not recommended [13], patients underwent relatively frequent imaging during our study. Nevertheless, of 19 patients who relapsed, only 2 were detected preclinically. This result is consistent with those of previous studies reporting $<10 \%$ detection of relapse in asymptomatic patients $[11,14]$. Regarding this result, there is skepticism not only about routine scanning but also LDH testing during posttreatment follow-up, as it may lead to additional radiological investigations, which are also unnecessary most of the time.

Although one may insist that $\mathrm{LDH}$ is a cost-effective test with high NPV, we should reflect on the fear and worry of patients who have presented with increased $\mathrm{LDH}$ levels and have received an explanation of the meaning 
from a physician. This is supported by a recent report indicating that surveillance CT scans are a source of anxiety and fear of recurrence in long-term lymphoma survivors [15]. Routine LDH testing may also have this type of adverse effect.

As for the noninvasive predictive or prognostic biomarkers of aggressive lymphoma, lymphopenia is now a promising candidate. Its prognostic value at the time of initial diagnosis has been reported [16-17], and its prognostic role following completion of first-line therapy has been demonstrated [18]. However, more detailed, larger studies should be conducted. Until such additional studies identify the ideal tumor marker for NHL, the best method for the surveillance of high-grade NHL after successful first-line treatment is a thorough physical examination with significant attention to the patient's symptoms. Routine serum LDH testing during posttreatment follow-up visits is of limited value and may create concern regarding the patient's fear and the cost of radiologic testing.

The retrospective nature of our analysis is a limitation of this study. However, the study does provide useful information for physicians regarding the issue of elevated $\mathrm{LDH}$ during and after treatment.

In conclusion, measuring serum LDH beyond initial diagnosis has no clear benefit in predicting disease progression during treatment or relapse after CR in patients with DLBCL treated with R-CHOP21 immunochemotherapy.

\section{References}

$\checkmark 1$ Coletta DF, Siegel PD: Lactic dehydrogenase. Med Sci 1964;15:98-101.

$\longrightarrow 2$ Schmoll HJ, Souchon R, Krege S, Albers P, Beyer J, Kollmannsberger C, Fossa SD, Skakkebaek NE, de Wit R, Fizazi K, Droz JP, Pizzocaro G, Daugaard G, de Mulder PH, Horwich A, Oliver T, Huddart R, Rosti G, Paz Ares L, Pont O, Hartmann JT, Aass N, Algaba F, Bamberg M, Bodrogi I, Bokemeyer C, Classen J, Clemm S, Culine S, de Wit M, Derigs HG, Dieckmann KP, Flasshove M, Garcia del Muro X, Gerl A, Germa-Lluch JR, Hartmann M, Heidenreich A, Hoeltl W, Joffe J, Jones W, Kaiser G, Klepp O, Kliesch S, Kisbenedek L, Koehrmann KU, Kuczyk M, Laguna MP, Leiva O, Loy V, Mason MD, Mead GM, Mueller RP, Nicolai N, Oosterhof GO, Pottek T, Rick O, Schmidberger H, Sedlmayer F, Siegert W, Studer U, Tjulandin S, von der Maase $\mathrm{H}$, Walz $\mathrm{P}$, Weinknecht $\mathrm{S}$, Weissbach L, Winter E, Wittekind C: European consensus on diagnosis and treatment of germ cell cancer: a report of the European Germ Cell Cancer Consensus Group (EGCCCG). Ann Oncol 2004;15:1377-1399.

-3 Bacci G, Avella M, McDonald D, Toni A, Orlandi M, Campanacci M: Serum lactate dehydrogenase (LDH) as a tumor marker in Ewing's sarcoma. Tumori 1988;74:649-655.

4 Gonzalez-Billalabeitia E, Hitt R, Fernandez J, Conde E, Martinez-Tello F, Enriquez de Salamanca R, Cortes-Funes H: Pre-treatment serum lactate dehydrogenase level is an important prognostic factor in high-grade extremity osteosarcoma. Clin Transl Oncol 2009;11: 479-483.

5 A predictive model for aggressive non-Hodgkin's lymphoma. The International NonHodgkin's Lymphoma Prognostic Factors Project. N Engl J Med 1993;329:987-994.

-6 Blay J, Gomez F, Sebban C, Bachelot T, Biron P, Guglielmi C, Hagenbeek A, Somers R, Chauvin F, Philip T: The International Prog- nostic Index correlates to survival in patients with aggressive lymphoma in relapse: analysis of the PARMA trial. Parma Group. Blood 1998;92:3562-3568.

7 Tilly H, Dreyling M: Diffuse large B-cell nonHodgkin's lymphoma: ESMO clinical recommendations for diagnosis, treatment and follow-up. Ann Oncol 2009;20(suppl 4):110-112.

$>8$ Coiffier B, Thieblemont C, Van Den Neste E, Lepeu G, Plantier I, Castaigne S, Lefort S, Marit G, Macro M, Sebban C, Belhadj K, Bordessoule D, Ferme C, Tilly H: Long-term outcome of patients in the LNH-98.5 trial, the first randomized study comparing rituximabCHOP to standard CHOP chemotherapy in DLBCL patients: a study by the Groupe d'Etudes des Lymphomes de l'Adulte. Blood 2010;116:2040-2045.

-9 Hong J, Park S, Park J, Kim HS, Kim KH, Ahn JY, Rim MY, Jung M, Sym SJ, Cho EK, Shin $\mathrm{DB}$, Lee JH: Evaluation of prognostic values of clinical and histopathologic characteristics in diffuse large B-cell lymphoma treated with rituximab, cyclophosphamide, doxorubicin, vincristine, and prednisolone therapy. Leuk Lymphoma 2011;52:1904-1912.

-10 Cheson BD, Pfistner B, Juweid ME, Gascoyne RD, Specht L, Horning SJ, Coiffier B, Fisher RI, Hagenbeek A, Zucca E, Rosen ST, Stroobants S, Lister TA, Hoppe RT, Dreyling M, Tobinai K, Vose JM, Connors JM, Federico M, Diehl V: Revised response criteria for malignant lymphoma. J Clin Oncol 2007;25: 579-586.

11 Weeks JC, Yeap BY, Canellos GP, Shipp MA: Value of follow-up procedures in patients with large-cell lymphoma who achieve a complete remission. J Clin Oncol 1991;9:11961203.

12 El-Sharkawi D, Basu S, Ocampo C, Qian W, D'Sa S, Hoskin PJ, Ardeshna KM: Elevated lactate dehydrogenase levels detected during routine follow-up do not predict relapse in patients with diffuse large B-cell lymphoma who achieve complete remission after primary treatment with rituximab, cyclophosphamide, doxorubicin, vincristine and prednisone-like immunochemotherapy. Leuk Lymphoma 2012;53:1949-1952.

13 Armitage JO: My treatment approach to patients with diffuse large B-cell lymphoma. Mayo Clin Proc 2012;87:161-171.

14 Guppy AE, Tebbutt NC, Norman A, Cunningham D: The role of surveillance CT scans in patients with diffuse large B-cell nonHodgkin's lymphoma. Leuk Lymphoma 2003;44:123-125.

15 Thompson CA, Charlson ME, Schenkein E, Wells MT, Furman RR, Elstrom R, Ruan J, Martin P, Leonard JP: Surveillance CT scans are a source of anxiety and fear of recurrence in long-term lymphoma survivors. Ann Oncol 2010;21:2262-2266.

16 Mitrovic Z, Perry AM, Suzumiya J, Armitage JO, Au WY, Coiffier B, Holte H, Jaffe ES, Monserrat E, Rajan SK, Savage KJ, Tobinai K, Vose JM, Weisenburger DD: The prognostic significance of lymphopenia in peripheral Tcell and natural killer/T-cell lymphomas: A study of 826 cases from the International Peripheral T-cell Lymphoma Project. Am J Hematol 2012;87:790-794.

17 Kim YR, Kim JS, Kim SJ, Jung HA, Kim WS, Lee HW, Eom HS, Jeong SH, Park JS, Cheong JW, Min YH: Lymphopenia is an important prognostic factor in peripheral T-cell lymphoma (NOS) treated with anthracyclinecontaining chemotherapy. J Hematol Oncol 2011;4:34.

18 Aoki T, Nishiyama T, Imahashi N, Kitamura $\mathrm{K}$ : Lymphopenia following the completion of first-line therapy predicts early relapse in patients with diffuse large $B$ cell lymphoma. Ann Hematol 2012;91:375-382. 